\title{
Rediscovering the musician's brain: a systematic review and meta-analysis
}

Criscuolo, Antonio ${ }^{1,2+}$, Pando-Naude, Victor ${ }^{2+*}$, Bonetti, Leonardo ${ }^{2}$, Vuust, Peter $^{2} \&$ Brattico, Elvira $^{2}$

${ }^{1}$ Department of Neuropsychology \& Psychopharmacology, Faculty of Psychology and Neuroscience, Maastricht University

${ }^{2}$ Center for Music in the Brain, Department of Clinical Medicine, Aarhus University \& The Royal Academy of Music Aarhus/Aalborg, Denmark

+Shared first-authorship

\section{*Corresponding author:}

Victor Pando-Naude, M.D., M.Sc., PhD Fellow

Center for Music in the Brain (MIB)

Department of Clinical Medicine, Aarhus University

Unversitetsbyen, 3-0-17

8000 Aarhus C, Denmark

Email: pandonaude@clin.au.dk

Phone: +4552805306 


\begin{abstract}
.
Musical expertise is a model of neuroplasticity associated with pervasive, long-lasting training effects. Indeed, decades of cognitive neuroscience widely investigated brain functional and structural changes associated with musical training, providing a widespread and variegated set of findings. However, several controversial results emerged, leading the neuroscientific community to lack a well-defined neuro-functional-anatomy of musical expertise. Here, we performed a systematic review and metaanalysis of publications investigating brain functional and structural differences between musicians and non-musicians. Eighty-four publications were included in the qualitative synthesis. Coordinate-based meta-analyses were conducted using the anatomic/activation likelihood estimation (ALE) method implemented in GingerALE, with a total of 675 foci, 79 experiments and 2780 participants. Results showed a widespread and complex array of functional and structural changes in musicians' brains, revealing for the first time a comprehensive picture of the brain plasticity associated with musical training.
\end{abstract}




\section{Introduction.}

A key to survival is the ability to adapt to the environment as biologically reflected in the neuroplastic changes associated with experience ${ }^{1}$. Thus, understanding the mechanisms behind neuroplasticity has always represented a primary and fascinating challenge in neuroscience ${ }^{2}$. At a circuit level, studies employing animal models showed how small neural networks are shaped by experience and learning, highlighting the role of key brain mechanisms such as long-term potentiation (LTP) and long-term depression (LTD) $)^{3}$.

Yet, the whole-brain neuroplasticity associated with the complexity of human life has remained more elusive and difficult to investigate. Studying the musician's brain has been suggested as an ideal model for tackling this complex challenge ${ }^{4}$, allowing for both cross-sectional and longitudinal investigations ${ }^{5,6}$.

Indeed, a long-lasting musical training promotes highly-specialized perceptual, motor, emotional and higher-order cognitive abilities ${ }^{7}$, as well as the capacity of integrating multimodal auditory, visual and motor information ${ }^{8}$. Musical training also requires the development of fine-grained skills in auditory perception, kinesthetic control, visual perception and pattern recognition ${ }^{9}$. Further, it stimulates mnemonic processes, fine-grained auditory perception (absolute pitch) ${ }^{10}$ and manual coordination. All these specialized abilities acquired through an extensive professional training have a neural counterpart, represented by a wide array of brain structural and functional neuroplastic changes associated with musical expertise.

In cognitive neuroscience, structural neuroplasticity is usually quantified in terms of decreased or increased volumes of gray and white matter (GM and WM, respectively) as measured by magnetic resonance imaging (MRI). GM volumes are typically derived from cortical thickness (CT) and voxelbased morphometry (VBM) analyses, and results are interpreted as differences in GM concentration ${ }^{11}$. WM volumes can be measured with VBM analysis as well, although the study of fractional anisotropy (FA) values obtained by diffusion tensor imaging (DTI) represents a valid and more informative alternative for investigating $\mathrm{WM}^{12}$. Differently, functional neuroplasticity can be examined in taskrelated studies by using metabolic measures of oxygen consumption as recorded by functional (f) MRI. Hence, the time-series of blood-oxygen-level-dependent (BOLD) signals are usually correlated to the tasks performed during image acquisition to infer region-specific or network-level activity patterns ${ }^{13}$.

Employing these techniques, decades of studies on musical expertise have provided a plethora of variegated findings which, while abundant, are scattered and often controversial. For instance, while some works reported an overall increase of GM volume when comparing musicians' to non-musicians' brains ${ }^{14,15}$, other research mainly highlighted negative correlations between GM volumes and musical expertise $^{16}$. Similarly, fMRI studies reported a large and sometimes non-consistent range of brain areas that were differently active during tasks when comparing musicians vs non-musicians ${ }^{17}$. Moreover, previous literature has mainly relied on cross-sectional comparisons rather than longitudinal designs, resulting in reduced consistency across studies and showing a lack of a well-defined neuro-functionalanatomy of musical expertise.

Strikingly, although the musician's brain has even been indicated as a "model of neuroplasticity", the neuroscientific community lacks meta-analytic efforts of summarizing the findings on brain differences between music experts and laypersons. Thus, for the first time, we conducted a coordinate-based metaanalysis of a large pool of MRI studies using state-of-the-art methods such as anatomic/activation likelihood estimation (ALE) ${ }^{18}$, to investigate the neuroanatomical and neurofunctional changes associated with musical training in healthy humans. 


\section{Results.}

A total of 1169 records was identified through database searching, and after removing duplicates, 679 records were initially screened by title and abstract. A total of 145 articles was assessed for eligibility in the full-text screening stage. From these, 84 studies fulfilled criteria for eligibility and were thus included in the qualitative synthesis. Finally, 58 studies reported results in stereotactic coordinates (either Talairach or Montreal Neurological Institute (MNI) three-dimensional-coordinate system) and were therefore included in the quantitative synthesis (ALE meta-analyses) (Supplementary Figure 1).

\subsection{Study characteristics.}

The characteristics of all studies included in our work are shown in Table 1. Eighty-four studies met inclusion criteria and were included in the qualitative synthesis. The total number of participants was 3048, with 1581 musicians (M), and 1424 non-musicians (NM). Eighteen studies (21\%) included amateur musicians, and only 7 studies $(8.3 \%)$ reported absolute pitch possessors $(n=97)$. Musical instruments were reported in the majority of the studies (81\%): piano or keyboard $(62 \%)$, string instruments $(41 \%)$, wind instruments $(26 \%)$, percussion instruments $(17 \%)$, voice $(8 \%)$, and $19 \%$ studies failed to report musicians' instrument. Years of education was described only in $8 \%$ of the included studies. Years of musical training was reported in $63 \%$ of the studies, with a mean of $15.6 \pm$ 5.9 years. The age of onset of musical training was reported in $49 \%$ of the studies, with a mean of 7.4 \pm 2.3 years old. Weekly hours of training were reported in $32 \%$ of the studies, with a mean of $16.7 \pm$ 8.9 hours per week.

Neuroimaging data was acquired in either $1.5 \mathrm{~T}(39 \%)$, or $3 \mathrm{~T}(56 \%)$ scanners, while $5 \%$ of studies did not report the magnetic field strength. MRI scanners included Siemens (40\%), General Electric (25\%), Philips (21\%), Bruker (5\%), while 7\% did not report it. Analysis methods included fMRI (52\%), VBM (29\%), DTI (18\%), and CT (10\%). Finally, $84 \%$ of studies included GM analyses, while $23 \%$ included WM analyses (Supplementary Tables 1 and 2).

\section{Insert Table 1.}

\subsection{MRI quality.}

MRI quality of the included studies in the meta-analysis was assessed following a set of guidelines for the standardized reporting of MRI studies (Supplementary Table 3). All studies reported their MRI design, software package and image acquisition, processing and analyses. Overall, good MRI practices were performed in the included studies.

\subsection{Primary outcome: ALE meta-analysis.}

The first ALE meta-analysis included all foci extracted from the screened studies (both functional and anatomical; 675 foci, 79 experiments, and 2,780 participants). The analysis returned significant clusters peaking in the following areas (alphabetical order): anterior cingulate cortex, caudate, claustrum, inferior frontal gyrus, insula, inferior parietal lobule, middle frontal gyrus, middle temporal gyrus, parahippocampal gyrus, postcentral gyrus, precentral gyrus, putamen, supramarginal gyrus, superior parietal lobule and superior temporal gyrus. The comparison $\mathrm{M}>\mathrm{NM}$ (higher volume/activity in musicians) resulted in significant peak clusters of both left and right superior temporal gyri, extending to middle temporal gyri, middle and inferior frontal gyri, insula and pre-post central gyri. The comparison $\mathrm{NM}>\mathrm{M}$ (lower volume/activity in musicians) resulted in significant peak clusters located in the superior and inferior parietal lobules, pre-post central gyri, caudate, and cerebellum (Figure 1,

Table 2). 
The comparison M vs NM was further analyzed for structural and functional foci, independently. Structurally, the comparison $\mathrm{M}>\mathrm{NM}$ in gray matter (GM) resulted in significant peak clusters of the superior temporal gyrus (BA22), including the Heschl's gyrus and planum temporale, postcentral gyrus, supramarginal gyrus, insula, hippocampus, thalamus, cerebellum and brainstem; and white matter (WM) tracts of the internal capsule bundle, bilaterally, corona radiata, corpus callosum, optic radiation and anterior thalamic radiations. Conversely, the comparison NM $>\mathrm{M}$ in GM resulted in significant peak clusters of the precentral gyrus, posterior cingulate cortex and supramarginal gyrus. No significant results were found in this comparison for WM. Functionally, $M>$ NM resulted in significant peak clusters of the inferior frontal gyrus, superior temporal gyrus, and middle frontal gyrus. The comparison $\mathrm{NM}>\mathrm{N}$ resulted in significant peak clusters of the inferior parietal lobule, precentral gyrus, superior frontal gyrus, superior parietal lobule and cerebellum (Figure 1, Supplementary Table 4).

Insert Figure 1.

Insert Table 2.

\subsection{Contrast analysis.}

A contrast ALE meta-analysis was conducted to compare foci from structural (e.g., VBM) and functional (e.g., fMRI) studies, by means of conjunction (similarity) and subtraction (difference). The purpose of this analysis was to identify common and distinct music-related brain areas between the neuroimaging modalities, thus, suggesting a more objective relationship between a function, and structural neuroadaptations produced by the repetition of that function. The superior temporal gyrus, precentral gyrus and insula are present in both modalities. The postcentral gyrus (BA3), supramarginal gyrus and transverse temporal gyrus are more present in structural imaging, while the inferior frontal gyrus, postcentral gyrus (BA4), middle frontal gyrus, middle temporal gyrus, and parietal lobules are more present in functional imaging (Figure 2, Supplementary Table 5).

\section{Insert Figure 2.}

\subsection{Subgroup analysis.}

A series of post hoc subgroup analyses were conducted to identify brain regions related to specific musical and cognitive features explored by the previous studies. Such features included improvisation, language, melody, memory, sensorimotor, pitch, rhythm and timbre (Figure 3, Supplementary Table 6).

The improvisation subgroup resulted in significant clusters of the right superior temporal gyrus extending to the insula, and precentral gyrus, bilaterally. Such results suggest the engagement of primary auditory and motor areas, presumably modulated by limbic processes. Motor-related foci converged in the precentral gyrus, inferior parietal lobule and medial frontal gyrus. Notably, motor, premotor and supplementary motor areas were expected. The language subgroup resulted in significant clusters in supramarginal gyrus, inferior parietal lobule, anterior cingulate cortex and middle frontal areas. Such areas suggest the activity of a network involved in language processing, and executive processing. Memory-related subgroup resulted in significant clusters in pre-postcentral gyri, middle, medial and superior frontal gyri. Interestingly, no areas from the parahippocampal gyrus were significant. The melody subgroup resulted in significant clusters of the cingulate gyrus, insula and superior temporal gyrus. Pitch subgroup resulted in convergence of foci in the insula extending to claustrum, inferior frontal gyrus and putamen. Rhythm-related foci converged in the supramarginal gyrus, angular gyrus, middle frontal gyrus, and somatosensory cortices. Timbre subgroup showed the inferior frontal gyrus, insula and inferior parietal lobule. Such physical auditory features in music seem 
to rely on the function of primary cortical areas for audition, somatosensation, language and emotion perception.

\section{Insert Figure 3.}

\subsection{Meta-analytic connectivity modelling (MACM) and functional characterization.}

Meta-analytic connectivity modelling (MACM) was performed to analyze co-activation patterns of the regions-of-interest (ROI) resulting from the primary outcome, aiming to functionally segregate each region's contribution to behavioral domains and paradigm classes. A total of 30 music expertise-related ROIs that resulted from the primary outcome were included. The ROIs were input into the BrainMap database separately, to identify studies that reported activation within each ROI boundary. Then, wholebrain coordinates of activations were downloaded as new foci files, and ALE meta-analyses were performed on the foci to identify areas of convergence. Finally, functional characterization histograms were used to categorize each area's participation in behavioral domains and paradigm classes (Supplementary Table 7 for MACM, and Supplementary Table 8 for functional characterization). 


\section{Discussion.}

Accumulating evidence has demonstrated that musical expertise is accompanied by several neuroanatomical and -functional changes, electing the long-lasting training of professional musicians as a privileged tool to investigate whole-brain neuroplasticity in humans. However, discrepancies between findings challenged our understanding of such phenomenon. Thus, here we provide for the first time a coordinate-based anatomic/activation likelihood estimation (ALE) meta-analysis aiming to depict a unified picture of the neuro-functional-anatomy of musical expertise. Besides confirming that music experts possess highly developed auditory, sensorimotor, and subcortical regions, we highlight convergence towards bilateral and widely distributed networks. Such results challenge the simplistic and most commonly accepted view of left-lateralization for music-related functions and structures. Rather, this meta-analysis strengthens the view that musical training is a beneficial complex and multisensory experience which stimulates a wide variety of cognitive functions.

\subsection{Characteristics of the included studies.}

In this systematic review and meta-analysis, the included publications reported a clear research question, inclusion and exclusion criteria for participants, description of methods and explicit results. Most of the studies used state-of-the-art techniques and computational MRI-tools, important for the support of standardization and reproducibility of neuroimaging studies. However, we found that some of the studies lacked important demographic data such as the years of education, age of musical training onset, and current time of musical practice, which may have an effect on behavioral tasks and neuroimaging data.

\subsection{Structural and functional neuroplasticity in musical expertise.}

Our results highlight that expert musicians exhibit higher GM volume in bilateral superior temporal gyri, postcentral gyrus, medial frontal gyrus, supramarginal gyrus, hippocampus, insula, thalamus and left cerebellum; and greater WM volume in bilateral internal capsule bundle, anterior corpus callosum, longitudinal fasciculus and anterior thalamic radiations as compared to non-musicians. Additionally, musicians exhibit higher activity of the inferior frontal gyrus and superior temporal gyrus related to auditory tasks. On the other hand, musicians exhibit lower GM volume in areas of the sensorimotor cortex and cingulate cortex. No WM structure was found to have larger volume in non-musicians as compared to musicians. Finally, musicians exhibit lower activation of the parietal lobes and cerebellum.

\section{The auditory system.}

One of our main findings shows enlargement of GM volume in musicians located in medial and posterior superior temporal regions, with clusters extending into primary and secondary auditory cortices. These regions include neuronal assemblies dedicated to encoding of spectro-temporal features of sounds relevant to music ${ }^{19}$, such as the discrete pitches forming the Western chromatic scale and fine changes in pitch intervals ${ }^{20}$. More specifically, it seems that the posterior supratemporal regions are more involved in encoding the height of pitch, whereas the anterior regions are representing the chroma, that is the pitch category irrespectively of the octave $\mathrm{e}^{21}$. Moreover, these areas participate in auditory imagery of melodies ${ }^{22}$ and in the processing of the contour and Gestalt patterns of melodies, allowing for recognition and mistakes discrimination ${ }^{23}$.

Beyond music-related functions, superior temporal regions are recruited for phonological processing and multimodal integration of sensory information. In particular, accumulating evidence has shown that STS and posterior STG, together with early auditory regions (HG), are involved for the processing of speech sounds, abstract representation of speech sounds and phonemes, and audiovisual integration 
mechanisms. Therefore, temporal regions seem to represent fundamental structures for both language and music processing ${ }^{24}$.

The inferior frontal gyrus has been described as an important hub of both the dorsal and ventral auditory streams. The dorsal auditory stream connects the auditory cortex with the parietal lobe, which projects in turn to the inferior frontal gyrus pars opercularis (Brodmann area 44). This area has been related to the articulatory network, dedicated to specific functions of speech comprehension and production, and highly connected to premotor and insular cortices ${ }^{25}$. The ventral auditory stream connects the auditory cortex with the middle temporal gyrus and temporal pole, which in turn connects to the inferior frontal gyrus pars triangularis (Brodmann area 45). This area has been associated with semantic processing ${ }^{26}$. These two regions within the inferior frontal gyrus constitute Broca's area.

\section{The parietal lobe.}

The parietal lobe has been also described as an integration area of sensory inputs. The superior parietal lobule includes Brodmann areas 5 and 7, which are involved in somatosensory processing and visuomotor coordination, respectively. The inferior parietal lobule includes Brodmann areas 39 and 40, the angular gyrus and supramarginal gyrus, respectively. The angular gyrus has been related to projection of visual information to Wernicke's area, memory retrieval and theory of $\operatorname{mind}^{27}$. The supramarginal gyrus is an integration hub of somatosensory input, and has been involved in language perception and processing ${ }^{28}$. Notably, these areas are also important relays in the dorsal auditory stream and the posterior part of the default mode network (DMN).

\section{The salience network.}

It has been proposed that the insula and the anterior cingulate cortex (ACC) form the salience network $(\mathrm{SN})$, that coordinates between the DMN and the central executive network $(\mathrm{CEN})^{29}$. The ACC has been related to cognitive and emotional processing. The cognitive component projects to prefrontal, motor and parietal areas to process top-down and bottom-up stimuli. The emotional component projects to amygdala, nucleus accumbens, hypothalamus, hippocampus, and insula, assessing the salience of emotional and motivational information ${ }^{30}$. The insula integrates information from the internal physiological state, and projects to the ACC, ventral striatum and prefrontal cortex to initiate adaptive responses ${ }^{31}$. Thus, enhanced function of these areas after musical training appears to result in a more efficient coordination between networks.

\section{The sensorimotor system.}

The precentral and postcentral gyrus represent the primary motor and somatosensory cortex, respectively. These two areas are divided by the central sulcus, whose extension represent the sensation and motion of segregated body parts. Our findings show both convergent and divergent effect of musical training in these areas, suggesting a more complex picture than previously thought. For example, neuroadaptations in the sensorimotor system may vary depending on the musical instrument of use $\mathrm{e}^{32}$.

The basal ganglia are nuclei of neurons important for the initiation and suppression of movements. In the motor loop of the basal ganglia, inputs from motor cortices project to the dorsal striatum, composed by the putamen and caudate. In the presence of adequate dopaminergic signaling, the direct pathway works to facilitate movement, while the indirect pathway suppresses it. By effective disinhibition processes, the striatum transiently inhibits the pallidum, and in turn, the motor area of the thalamus is disinhibited and free to project back to the motor cortex and initiate a motor program down the corticospinal tract. Similarly, the subthalamic nucleus in the indirect pathway is transiently inhibited when suppressing movement, increasing the inhibition of the pallidum over the thalamus, therefore 
blocking the motor cortex activity ${ }^{33}$. Our findings show neuroadaptive processes in the putamen and caudate of musicians, presumably resulting in enhancement of such disinhibition mechanisms reflected in fine movement control.

The cerebellum has been shown to play a crucial role in multiple cognitive processes such as sensory discrimination, rhythmic perception and production, working memory, language, and cognition ${ }^{34}$. Previous fMRI studies in humans suggest that the cerebellum shows segregated activations for motor and cognitive tasks. Motor tasks seem to activate lobules IV-VI in the superior parts of the anterior cerebellum. In contrast, attentional or working memory tasks activate posterior cerebellar hemispheres, namely lobule VIIA, which is divided to crus I and crus II, as well as lobule VIIB ${ }^{35}$. Musicians and nonmusicians show GM volume differences in the cerebellum, specifically in area Crus I, as reported in our results. It has been demonstrated that the activity in crus I/II has a specific relationship with cognitive performance and is linked with lateral prefrontal areas activated by cognitive load increase ${ }^{36}$. In other words, the crus I/II seems to optimize the response time when the cognitive load increases. Additionally, it has been suggested that crus I/II is associated with beat discrimination thresholds. Thus, there is a positive correlation between GM volume in crus I and beat discrimination performance, evidenced by enhanced ability in musicians ${ }^{37}$.

\section{White matter.}

With regards to WM, we found significant clusters comparing M vs NM that were located in the WM tracts of internal capsule, corpus callosum, longitudinal fasciculus, and thalamic radiations. The internal capsule is a WM structure which connects basal ganglia regions and carries information from and to surrounding cerebral cortex. Connecting fibers in basal ganglia might be thickened by musical expertise because of their involvement in motor control, rhythmic processing, sequence learning, reinforcement learning and memory processes ${ }^{38}$. In general, basal ganglia structures are recruited during working memory processing for musical motifs ${ }^{39}$ and the most ventral regions are a core structure of the reward circuit. Interestingly, they are found to be more active in musicians as compared to non-musicians while listening to expressive music ${ }^{40}$.

Lastly, the observed increases in the $\mathrm{CC}$ volume may be related to the facilitation of necessary communications between hemispheres and functional networks underlying the coordination of complex sequential bimanual motor sequences and the recall of stored motor programs ${ }^{41}$.

\subsection{Limitations and future perspectives.}

This comprehensive review had the scope to provide a unified synthesis of the effects of musical training on brain structure and functions. Our qualitative review further highlights that previous studies in this field are characterized by heterogeneity of methods, paradigms, and sample backgrounds, as well as relevant missing information. While arguing that the field will benefit from more clarity (e.g. thorough description of methods) and consistency across future studies, we also delineate limitations for our meta-analysis. For example, we set a contrast based on the comparison M vs NM with the aim to narrow down the heterogeneity of the sample and methods used. However, by doing so we relied on two assumptions: (1) the data we pool is based on best research practices; (2) the validity of the GingerALE method. Indeed, to conduct the ALE meta-analysis, we pooled peak coordinates derived from the included studies, rather than using original raw structural MRI images. Thus, the accuracy of our findings relies on the result of a statistical estimation of coordinate-based anatomic foci (input), treated as spatial probability distributions centered at the given coordinates. The heterogeneity of the methods in use in previous studies (ranging from preprocessing software, smoothing, statistical thresholds and participants' characteristics) are not under our control and represent potential 
confounders for the results. Perhaps a regression-based assessment of the influence of those heterogenous factors on the findings would sharpen the results. However, meta-regression analysis is not compatible with GingerALE. When assessing publication bias, all clusters resulting from the primary outcome showed an FSN greater than the minimum imposed of 17, indicating a robust convergence of foci in these regions (further information is reported in Supplementary Table 9).

Lastly, on a more theoretical perspective, our results contribute but do not solve the long-standing "nature vs nurture" debate. Indeed, based on evidence that musical training stimulates higher-cognitive functions, auditory-motor integration, attention, memory and engages reward networks, some have suggested that it may be particularly effective in driving neuroplastic mechanisms ${ }^{42}$. However, we are indeed blind to whether the highlighted differences emerging when comparing M Vs NM are trainingdependent or due to innate predispositions. In line with the above-mentioned view there are multiple studies demonstrating a strong correlational link between the length of musical training and neurofunctional-anatomical changes. The idea of use-dependent structural changes is further supported by the study conducted by Gaser \& Schlaug ${ }^{43}$ which reported that amateur musicians showed an intermediate increase in gray matter volume when compared to NM and M. Interestingly, the same pattern was found when comparing cognitive abilities, with amateurs showing higher cognitive abilities than NM, but lower than $\mathrm{M}^{44}$. Lastly, beyond the statistical power of common inferential studies, a longitudinal neuroanatomical study conducted by Hyde et al. ${ }^{45}$ in children, provided the most compelling evidence in support of the association between musical training and neuroplastic effects. The study showed that, despite the absence of behavioral or brain differences at baseline (prior to any training) in children assigned to musical training and control groups, structural brain changes were visible after only 15 months of musical training. In particular, they found increases in the volume of corpus callosum, auditory and motor cortices and these group differences correlated with improvements in musically relevant motor and auditory skills.

Altogether, the most reasonable conclusion is that the observed neuro-anatomical and -functional changes may be attributed to the interaction between brain maturation processes and the environmental demands of musical training ${ }^{13,43,50}$. Only when a consistent amount of longitudinal studies will be published, future systematic reviews and meta-analyses will be able to determine the causal link between musical training and brain changes. 


\section{Conclusions.}

The musician's brain has been repeatedly suggested as an ideal example of neuroplasticity. Yet, decades of cognitive neuroscience have provided a scattered and partially controversial series of findings. Thus, with the present comprehensive coordinate-based meta-analysis, we have made a first necessary attempt to provide a unified quantitative and qualitative synthesis of the link between musical training and neuro-anatomical and -functional changes. Results highlight that music experts possess highly developed auditory, sensorimotor and subcortical regions. Indeed, we show that music experts exhibit bilateral widespread neuro-anatomical and functional differences as compared to laypersons, challenging the previous simplistic perspective focused on the left-lateralization of the brain for musicrelated functions. Conversely, these findings contribute to the notion that musical training is a complex multisensory experience and cannot be anchored to a few numbers of specialized brain regions only.

In conclusion, our findings rediscover the musician's brain and refine the knowledge related to its neuroplasticity. On top of this, we propose that long-lasting professional trainings represent remarkable resources to gain insights on the neuroplastic mechanisms that regulate the human brain. 


\section{Methods.}

\subsection{Literature search, screening and extraction.}

This systematic review and meta-analysis followed procedures from the Cochrane Handbook for Systematic Reviews ${ }^{48}$ and from the Centre for Reviews and Dissemination (Centre for Reviews and Dissemination, 2014). The review protocol was registered with PROSPERO No. [CRD42017060365]. This review was carried in accordance with the PRISMA statement ${ }^{49}$.

\subsubsection{Search strategy.}

Systematic search was performed using PubMed, PsycInfo and Scopus, of publications that reported brain structural or functional differences between M and NM. The search (August, 2020) included MeSH terms ("music", "education", "brain", "motor skills", "magnetic resonance imaging") and key words ("musical training", "musician"). No years or places of publication were imposed.

\subsubsection{Study eligibility.}

For qualitative synthesis, studies were included if they met the following criteria: (1) studies comparing brain structure and function between musicians and non-musicians, (2) in adult population, (3) by means of magnetic resonance imaging, in either structural modality (e.g., voxel-based morphometry [VBM]) or functional modality (e.g., functional magnetic resonance imaging[fMRI]). For quantitative synthesis (meta-analysis), studies were included if results were reported in stereotactic coordinates either Talairach or Montreal Neurological Institute (MNI) three-dimensional-coordinate system.

Studies were excluded using the following criteria: (1) review articles with no original experimental data, (2) neuroimaging data from non-MRI studies (e.g. PET), (3) pathological population, (4) longitudinal designs, (5) functional connectivity analyses, and (6) analyses were region-of-interest (ROI)-based and not whole-brain based (only quantitative synthesis).

Two reviewers (AC and VP) independently screened by title and abstract and selected articles for fulltext review, and also performed full-text reviews. Screening and data extraction were performed using the Covidence tool ${ }^{50}$. Any disagreements that arose between the reviewers were resolved through discussion or by a third and/or fourth reviewer (LB, EB).

\subsubsection{Data extraction.}

From each study, the following variables were extracted: first author, year of publication, population of interest, number of participants, age, sex, absolute pitch, musical feature, years of education, years of musical training, age of musical training onset, weekly training, musical instrument, MRI-system, MRImodel, head-coil, image acquisition parameters of T1, T2* and DWI sequences, repetition time (TR), echo time (TE), voxel size, analysis method and software. The main outcome to extract was any difference in structure or function, in stereotactic coordinates, comparing a musician group and a nonmusician group. If any of these points were not reported in the original article, authors were contacted to retrieve this information. Six authors were contacted, with 2 positive answers.

\subsection{Quality assessment of MRI studies.}

Criteria for MRI quality reporting was selected from a set of guidelines for the standardized reporting of MRI studies ${ }^{51,52}$. Such guidelines dictate a more consistent and coherent policy for the reporting of MRI methods to ensure that methods can be understood and replicated. 


\subsection{Activation likelihood estimation (ALE) and meta-analytic connectivity modelling (MACM).}

To test the convergence of findings from the neuroimaging studies, we used the anatomic/activation likelihood estimation (ALE) method implemented in the GingerALE software v3.0.2 $2^{18}$, a widely used technique for coordinate-based meta-analysis of neuroimaging data. Statistically significant foci from between-group contrasts were extracted and recorded for each study. If necessary, coordinates were converted from Talairach coordinates to MNI space using the Lancaster transform (icbm2tal) incorporated in GingerALE ${ }^{53,54}$. The ALE method treats activation foci (input) not as single points, but as spatial probability distributions centered at the given coordinates. Therefore, the algorithm tests to what extent the spatial locations of the foci correlate across independently conducted MRI studies investigating the same construct and assesses them against a null-distribution of random spatial association between experiments ${ }^{46}$. Statistical significance of the ALE scores was determined by a permutation test using cluster-level inference at $\mathrm{p}<0.05$ (FWE). As we did not impose any minimum cluster size of supra-threshold voxels, small volume clusters should be interpreted with caution.

First, the primary outcome was to identify brain structural and functional differences measured by MRI between musicians (M) and non-musicians (NM), pooling all coordinates (foci) together, to examine comprehensively the neural signatures of musical expertise. To test the directionality of the primary outcome, foci were pooled reporting higher volume/activity in musicians $(M>N M)$ and lower volume/activity in musicians $(\mathrm{NM}>\mathrm{M})$. Second, we conducted a contrast analysis to test for similarity (conjunction) and difference (subtraction), between the results of functional and structural foci, independently. Finally, a series of post hoc subgroup analyses were conducted to identify brain regions related to specific musical features such as: improvisation, language, melody, memory, motor, pitch, rhythm and timbre.

Meta-analytic connectivity modelling (MACM) was performed to analyze co-activation patterns of regions-of-interest (ROI) resulting from the primary outcome, aiming to functionally segregate each region's putative contribution to behavioral domains ${ }^{55,56}$. Co-activation analyses were performed using Sleuth ${ }^{57}$ and GingerALE from the BrainMap platform. To identify regions of significant convergence, an ALE meta-analysis was performed over all foci retrieved after searching Sleuth by each musicrelated ROI independently, and included the experiment level search criteria of "context: normal mapping" and "activations: activation only". Music-related ROIs were created in Mango ${ }^{58}$ with a $5 \mathrm{~mm}$ radius sphere. The functional characterization of music-related ROIs was based on the "Behavioral Domain" (BD) meta-data categories available for each neuroimaging study in the BrianMap database which include action, perception, emotion, cognition and interoception.

All meta-analytic results (ALE maps) were visualized using Mango on the MNI152 1mm standard brain, and resulting coordinates were cross-referenced to the Harvard-Oxford Cortical and Subcortical Atlas and the Juelich Histological Atlas via NeuroVault ${ }^{59}$ and FSLeyes ${ }^{60}$, respectively.

\subsection{Fail-Safe $N$ analysis (FSN).}

Finally, as traditional meta-analyses, coordinate-based meta-analyses such as ALE can be subject to different forms of publication bias which may impact results and invalidate findings (e.g., the "file drawer problem"). Thus, the Fail-Safe $\mathrm{N}$ analysis (FSN $)^{61}$ was performed as a measure of robustness against potential publication bias. It refers to the amount of contra-evidence that can be added to a metaanalysis before the results change, and can be obtained for each cluster that survives thresholding in an ALE meta-analysis. For normal human brain mapping, it is estimated that a $95 \%$ confidence interval for the number of studies that report no local maxima varies from 5 to 30 per 100 published studies. 
Using the upper bound and the fact that the present ALE meta-analysis consists of 58 studies, an estimate for the number of unpublished studies is 17. Therefore, the minimum FSN was defined as 17. A higher FSN indicates more stable results and hence a higher robustness. Such analyses were conducted only on the primary outcome. 


\section{Acknowledgments.}

The Center for Music in the Brain (MIB) is supported by the Danish National Research Foundation (grant number DNRF 117). The authors wish to thank Hella Kastbjerg for assistance with language check and proof reading. 


\section{Authors' contributions.}

AC, VPN and EB designed the meta-analysis. VPN guided AC and LB in the initial steps of the metaanalysis after which AC and LB conducted the screening of the studies, and VPN controlled the final selection. AC performed the sensitivity analysis, wrote the first draft of the manuscript and prepared the initial versions of the tables. EB and LB edited and wrote paragraphs in the Introduction and Discussion, whereas VPN wrote paragraphs in the Methods, Results and Discussion sections. VPN prepared the figures and tables. PV contributed to financially supporting the study and commented the initial versions of the manuscripts. EB finalized the paper for submission and all authors edited the manuscript and approved its final version. 
bioRxiv preprint doi: https://doi.org/10.1101/2021.03.12.434473; this version posted March $12,2021$. The copyright holder for this preprint

(which was not certified by peer review) is the author/funder, who has granted bioRxiv a license to display the preprint in perpetuity. It is made available under aCC-BY-NC-ND 4.0 International license.

\section{Conflict of interest.}

There is no actual or potential financial and other conflict of interest related to this manuscript. 


\section{References}

1. Wolf, C. \& Linden, D. E. J. Biological pathways to adaptability-interactions between genome, epigenome, nervous system and environment for adaptive behavior On the origin of adaptability. Genes, Brain Behav. 11, 3-28 (2012).

2. Jäncke, L. The plastic human brain. Restorative Neurology and Neuroscience 27, 521-538 (2009).

3. Lüscher, C. \& Malenka, R. C. NMDA receptor-dependent long-term potentiation and longterm depression (LTP/LTD). Cold Spring Harb. Perspect. Biol. 4, 1-15 (2012).

4. Münte, T. F., Altenmüller, E. \& Jäncke, L. The musician's brain as a model of neuroplasticity. Nat. Rev. Neurosci. 3, 473-478 (2002).

5. Bermudez, P. \& Zatorre, R. Differences in Gray Matter between Musicians and Nonmusicians. Ann. N. Y. Acad. Sci. 1060, 395-399 (2005).

6. Wollman, I., Penhune, V., Segado, M., Carpentier, T. \& Zatorre, R. J. Neural network retuning and neural predictors of learning success associated with cello training. PNAS Proc. Natl. Acad. Sci. United States Am. 115, E6056-E6064 (2018).

7. Reybrouck, M. et al. Music and and Brain Brain Plasticity : Music Plasticity : How How Sounds Sounds Trigger Trigger Neurogenerative Adaptations Neurogenerative Adaptations. doi:10.5772/intechopen. 74318

8. Pantev, C. \& Herholz, S. C. Plasticity of the human auditory cortex related to musical training. Neuroscience and Biobehavioral Reviews (2011). doi:10.1016/j.neubiorev.2011.06.010

9. Barrett, K. C., Ashley, R., Strait, D. L. \& Kraus, N. Art and science: how musical training shapes the brain. Front. Psychol. (2013). doi:10.3389/fpsyg.2013.00713

10. Schlaug, G. The brain of musicians. A model for functional and structural adaptation. Ann. N. Y. Acad. Sci. 930, 281-299 (2001).

11. Ashburner, J. \& Friston, K. J. Voxel-Based Morphometry-The Methods. (2000). doi:10.1006/nimg.2000.0582

12. Alexander, A. L., Lee, J. E., Lazar, M. \& Field, A. S. Diffusion Tensor Imaging of the Brain. (2007).

13. Glover, G. H. Overview of functional magnetic resonance imaging. Neurosurgery Clinics of North America 22, 133-139 (2011).

14. Vaquero, L. et al. Structural neuroplasticity in expert pianists depends on the age of musical training onset. Neuroimage 126, 106-119 (2016).

15. James, C. E. et al. Musical training intensity yields opposite effects on grey matter density in cognitive versus sensorimotor networks. Brain Struct. Funct. 219, 353-366 (2014).

16. Baer, L. H. et al. Regional cerebellar volumes are related to early musical training and finger tapping performance. Neuroimage 109, 130-139 (2015).

17. Clayton, K. K. et al. Executive function, visual attention and the cocktail party problem in musicians and non-musicians. PLoS One 11, 17 (2016).

18. Eickhoff, S. B. et al. Coordinate-Based Activation Likelihood Estimation Meta-Analysis of Neuroimaging Data: A Random-Effects Approach Based on Empirical Estimates of Spatial Uncertainty. (2009). doi:10.1002/hbm.20718

19. Schneider, P. et al. Structural and functional asymmetry of lateral Heschl's gyrus reflects pitch perception preference. Nat. Neurosci. 8, 1241-1247 (2005). 
20. Brattico, E., Tervaniemi, M., Näätänen, R. \& Peretz, I. Musical scale properties are automatically processed in the human auditory cortex. Brain Res. 1117, 162-174 (2006).

21. Warren, J. D., Uppenkamp, S., Patterson, R. D. \& Griffiths, T. D. Analyzing Pitch Chroma and Pitch Height in the Human Brain. in Annals of the New York Academy of Sciences 999, 212 214 (2003).

22. Zatorre, R. J. There's more to auditory cortex than meets the ear. Hear. Res. 229, 24-30 (2007).

23. Hyde, K. L., Peretz, I. \& Zatorre, R. J. Evidence for the role of the right auditory cortex in fine pitch resolution. Neuropsychologia 46, 632-639 (2008).

24. Peretz, I., Vuvan, D., Lagrois, M.-É. \& Armony, J. L. Neural overlap in processing music and speech. Philos. Trans. R. Soc. B 370, 20140090 (2015).

25. Brown, S., Martinez, M. J. \& Parsons, L. M. Music and language side by side in the brain: a PET study of the generation of melodies and sentences. Eur. J. Neurosci. 23, 2791-2803 (2006).

26. DeWitt, I. \& Rauschecker, J. P. Phoneme and word recognition in the auditory ventral stream. Proc. Natl. Acad. Sci. U. S. A. 109, 2709 (2012).

27. Seghier, M. L. The angular gyrus: Multiple functions and multiple subdivisions. Neuroscientist 19, 43-61 (2013).

28. Hartwigsen, G. et al. Phonological decisions require both the left and right supramarginal gyri. Proc. Natl. Acad. Sci. U. S. A. 107, 16494-16499 (2010).

29. Menon, V. \& Uddin, L. Q. Saliency, switching, attention and control: a network model of insula function. Brain structure \& function 214, 655-667 (2010).

30. Bush, G., Luu, P. \& Posner, M. I. Cognitive and emotional influences in anterior cingulate cortex. Trends in Cognitive Sciences 4, 215-222 (2000).

31. Brown, S., Martinez, M. J. \& Parsons, L. M. Passive music listening spontaneously engages limbic and paralimbic systems. Neuroreport 15, 2033-2037 (2004).

32. Schneider, P., Sluming, V., Roberts, N., Bleeck, S. \& Rupp, A. Structural, functional, and perceptual differences in Heschl's gyrus and musical instrument preference. Ann. N. Y. Acad. Sci. 1060, 387-394 (2005).

33. Purves, D. et al. Neuroscience 5th. Edition. (Sinauer Associates, Inc., Sunderland., 2012).

34. Kim, S., Ugurbil, K. \& Strick, P. Activation of a cerebellar output nucleus during cognitive processing. Science (80-. ). 265, 949-951 (1994).

35. Desmond, J. E., Chen, S. H. A. \& Shieh, P. B. Cerebellar Transcranial Magnetic Stimulation Impairs Verbal Working Memory. (2005). doi:10.1002/ana.20604

36. Salmi, J. et al. Cognitive and Motor Loops of the Human Cerebro-cerebellar System. J. Cogn. Neurosci. 22, 2663-2676 (2010).

37. Grahn, J. A. \& Brett, M. Rhythm and Beat Perception in Motor Areas of the Brain. J. Cogn. Neurosci. 19, 893-906 (2007).

38. Lehericy, S. et al. Distinct basal ganglia territories are engaged in early and advanced motor sequence learning. Proc. Natl. Acad. Sci. 102, 12566-12571 (2005).

39. Koelsch, S. et al. Functional architecture of verbal and tonal working memory: An fMRI study. Hum. Brain Mapp. 30, 859-873 (2009). 
40. Brattico, E. et al. It's sad but I like it: The neural dissociation between musical emotions and liking in experts and laypersons. Front. Hum. Neurosci. 9, 21 (2016).

41. Burunat, I. et al. Action in perception: Prominent visuo-motor functional symmetry in musicians during music listening. PLoS One 10, (2015).

42. Steele, C. J., Bailey, J. A., Zatorre, R. J. \& Penhune, V. B. Early musical training and whitematter plasticity in the corpus callosum: evidence for a sensitive period. J Neurosci 33, 1282 1290 (2013).

43. Gaser, C. \& Schlaug, G. Brain Structures Differ between Musicians and Non-Musicians. (2003).

44. Criscuolo, A., Bonetti, L., Särkämö, T., Kliuchko, M. \& Brattico, E. On the Association Between Musical Training, Intelligence and Executive Functions in Adulthood. Front. Psychol. 10, 1704 (2019).

45. Hyde, K. L. et al. The effects of musical training on structural brain development: A longitudinal study. Ann. N. Y. Acad. Sci. 1169, 182-186 (2009).

46. Hudziak, J. J. et al. Cortical thickness maturation and duration of music training: Healthpromoting activities shape brain development. J. Am. Acad. Child Adolesc. Psychiatry 53, 1153-1161.e2 (2014).

47. Bailey, J. A., Zatorre, R. J. \& Penhune, V. B. Early Musical Training is lnked to gray matter Structure in the Ventral Premotor Cortex and Auditory-motor Rhythm Sybcrhonization performance. J. Neurosci. 26, 755-767 (2014).

48. Higgins, J. P. \& Green, S. Cochrane Handbook for Systematic Reviews of Interventions. (2011).

49. Moher, D., Liberati, A., Tetzlaff, J., Altman, D. G. \& Group, T. P. Preferred Reporting Items for Systematic Reviews and Meta-Analyses: The PRISMA Statement. 6, 1-5 (2009).

50. Covidence systematic review software, Veritas Health Innovation, Melbourne, Australia. Available at www.covidence.org. Available at: https://support.covidence.org/help/how-can-icite-covidence.

51. Poldrack, R. A. et al. Guidelines for reporting an fMRI study. Neuroimage 40, 409-414 (2008).

52. Nichols, T. E. et al. Best practices in data analysis and sharing in neuroimaging using MRI. Nat. Neurosci. 20, 299-303 (2017).

53. Eickhoff, S. B., Bzdok, D., Laird, A. R., Kurth, F. \& Fox, P. T. Activation likelihood estimation meta-analysis revisited. Neuroimage 59, 2349-2361 (2012).

54. Laird, A. R. et al. Comparison of the disparity between Talairach and MNI coordinates in functional neuroimaging data: Validation of the Lancaster transform. (2010). doi:10.1016/j.neuroimage.2010.02.048

55. Laird, A. R. et al. Networks of task co-activations. Neuroimage 80, 505-514 (2013).

56. Robinson, J. L., Laird, A. R., Glahn, D. C., Lovallo, W. R. \& Fox, P. T. Metaanalytic Connectivity Modeling: Delineating the Functional Connectivity of the Human Amygdala. Hum. Brain Mapp. 31, 173-184 (2010).

57. Laird, A. R., Lancaster, J. L. \& Fox, P. T. BrainMap: The Social Evolution of a Human Brain Mapping Database. Neuroinformatics (2005).

58. Lancaster, J. L. et al. Automated regional behavioral analysis for human brain images. Front. 
Neuroinform. 6, 1-12 (2012).

59. Gorgolewski, K. J. et al. NeuroVault.org: a web-based repository for collecting and sharing unthresholded statistical maps of the human brain. Front. Neuroinform. 9, 1-9 (2015).

60. Jenkinson, M., Beckmann, C. F., Behrens, T. E. J., Woolrich, M. W. \& Smith, S. M. FSL. Neuroimage 62, 782-790 (2012).

61. Acar, F., Seurinck, R., Eickhoff, S. B. \& Moerkerke, B. Assessing robustness against potential publication bias in Activation Likelihood Estimation (ALE) meta-analyses for fMRI. PLoS One 13, e0208177 (2018). 


\section{Figure legends.}

Figure 1. Anatomic likelihood estimation meta-analytic results for studies comparing brain structure and function between $M$ and $N M$ at cluster level inference $p<0.05$ (FWE). The primary outcome included ALE meta-analysis of all included foci, and the contrast M vs NM for structural and functional modalities, independently. $\mathrm{M}>\mathrm{NM}=$ higher volume/activity in musicians; $\mathrm{NM}<\mathrm{NM}=$ lower volume/activity in musicians. Z, peak Z-value.

Figure 2. Conjunction analyses comparing foci from structural (CT, DTI, VBM) and functional (fMRI) studies, at cluster level inference p $<0.05$ (FWE). Contrast analyses were performed for structural and functional studies, and were tested for similarity (conjunction) and difference (subtraction), to illustrate common and/or distinct areas between the neuroimaging modalities. ALE, anatomic likelihood estimation value; Z, peak Z-value.

Figure 3. Musical feature subgroup meta-analyses, at cluster level inference $p<0.05$ (FWE). Z, peak Z-value. 


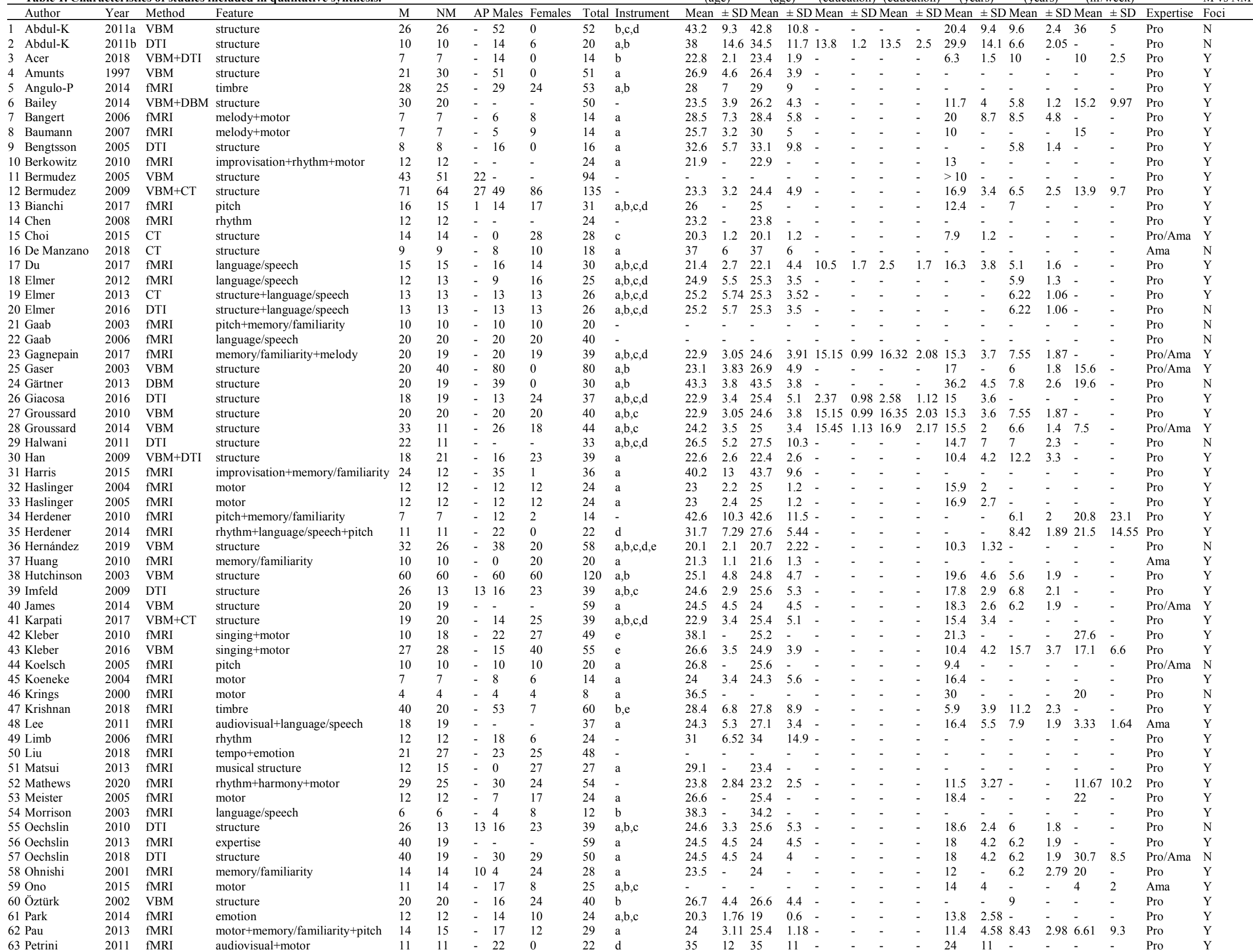


$\begin{array}{lllllllllllllllllllll}24.7 & 5.1 & 26.5 & 2.5 & - & - & - & - & - & - & 6.5 & 2.3 & 3.3 & 2.5 & \text { Pro/Ama } & \mathrm{N}\end{array}$ $\begin{array}{lllllllllllllllllll}20.8 & 2.3 & 20.4 & 1.5 & - & - & - & - & 11.6 & 3.4 & - & - & 10.9 & - & \text { Pro }\end{array}$

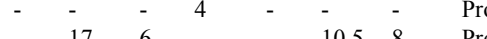
$\begin{array}{lllllllllllllll}26.1 & 3.8 & 26.5 & 4.6 & - & - & - & - & - & - & - & - & - & - & \text { Pro }\end{array}$ $\begin{array}{llllllllllllllllll}26.1 & 3.8 & 26.5 & 4.6 & - & - & - & - & - & - & - & - & - & - & - & - & \text { Pro }\end{array}$ $31.2 \quad 11.2 \quad 42.2 \quad 20.1$

$\begin{array}{llll}43.2 & 9.3 & 42.8 & 10.8\end{array}$

$\begin{array}{llll}42.2 & 10.1 & 40.1 & 11.8\end{array}$

$\begin{array}{llll}22.7 & 4.14 & 26.4 & 4.71 \\ 24.9 & 4.86 & 24.1 & 4.39\end{array}$ $\begin{array}{lllllllllllllllll}23.1 & 4.63 & 25 & 4.25 & 14.03 & 3.08 & 14.06 & 2.83 & 15.7 & 4.26 & 7.19 & 2.39 & - & - & \text { Pro }\end{array}$

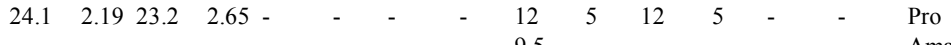

M, musicians; NM, non-musicians; CT, cortical thickness; DBM, deformation-l 
Table 2. Anatomic likelihood estimation meta-analytic results for studies comparing brain structure and function between $M$ and NM at cluster level inference $\mathbf{p}<\mathbf{0 . 0 5}$ (FWE).

\begin{tabular}{|c|c|c|c|c|c|c|c|c|}
\hline \multirow[t]{2}{*}{ Cluster number } & \multirow{2}{*}{$\begin{array}{l}\text { Volume } \\
\left(\mathrm{mm}^{3}\right)\end{array}$} & \multicolumn{3}{|c|}{ MNI coordinates } & \multirow[t]{2}{*}{ ALE } & \multirow[t]{2}{*}{$\mathrm{P}$} & \multirow[t]{2}{*}{$\mathrm{Z}$} & \multirow[t]{2}{*}{ Label (Side region BA) } \\
\hline & & $\mathrm{x}$ & $\mathrm{y}$ & $\mathrm{z}$ & & & & \\
\hline \multicolumn{9}{|c|}{ 1. All foci: 675 foci, 79 experiments, 2780 subjects } \\
\hline \multirow[t]{47}{*}{1} & 42464 & 50 & 10 & 22 & $3 \mathrm{E}-02$ & $3 \mathrm{E}-07$ & 5.0 & R Inferior Frontal Gyrus BA9 \\
\hline & & 54 & -10 & 4 & $3 \mathrm{E}-02$ & $1 \mathrm{E}-06$ & 4.7 & R Superior Temporal Gyrus BA22 \\
\hline & & 54 & 24 & 10 & $3 \mathrm{E}-02$ & 2E-06 & 4.6 & R Inferior Frontal Gyrus BA45 \\
\hline & & 50 & -32 & 18 & $3 E-02$ & $5 E-06$ & 4.4 & R Superior Temporal Gyrus BA41 \\
\hline & & 64 & -14 & 0 & $2 \mathrm{E}-02$ & $1 \mathrm{E}-05$ & 4.2 & R Superior Temporal Gyrus BA22 \\
\hline & & 54 & 12 & 0 & $2 \mathrm{E}-02$ & 2E-04 & 3.6 & R Precentral Gyrus BA44 \\
\hline & & 60 & -44 & -6 & $2 \mathrm{E}-02$ & $3 \mathrm{E}-04$ & 3.4 & R Middle Temporal Gyrus BA21 \\
\hline & & 62 & -8 & -8 & $2 \mathrm{E}-02$ & $3 \mathrm{E}-04$ & 3.4 & R Middle Temporal Gyrus BA21 \\
\hline & & 28 & 2 & -4 & 2E-02 & 8E-04 & 3.1 & R Putamen \\
\hline & & 58 & -36 & 24 & $2 \mathrm{E}-02$ & 9E-04 & 3.1 & R Insula BA13 \\
\hline & & 58 & -2 & -6 & $2 \mathrm{E}-02$ & 1E-03 & 3.1 & R Superior Temporal Gyrus BA22 \\
\hline & & 50 & -28 & -4 & $2 \mathrm{E}-02$ & $1 \mathrm{E}-03$ & 3.1 & R Insula BA22 \\
\hline & & 32 & 22 & -10 & $2 \mathrm{E}-02$ & $1 \mathrm{E}-03$ & 3.1 & R Insula BA47 \\
\hline & & 58 & 2 & 34 & $2 \mathrm{E}-02$ & $1 \mathrm{E}-03$ & 3.0 & R Precentral Gyrus BA6 \\
\hline & & 52 & -44 & 36 & $2 \mathrm{E}-02$ & $2 \mathrm{E}-03$ & 2.9 & R Supramarginal Gyrus BA40 \\
\hline & & 52 & -62 & -4 & $1 \mathrm{E}-02$ & 2E-03 & 2.9 & R Inferior Temporal Gyrus BA19 \\
\hline & & 54 & -60 & -8 & $1 \mathrm{E}-02$ & 3E-03 & 2.7 & R Middle Occipital Gyrus BA19 \\
\hline & & 36 & 22 & 8 & $1 \mathrm{E}-02$ & $3 \mathrm{E}-03$ & 2.7 & R Insula BA13 \\
\hline & & 66 & -40 & 8 & $1 \mathrm{E}-02$ & 7E-03 & 2.4 & R Middle Temporal Gyrus BA22 \\
\hline & & 56 & -10 & 22 & $1 \mathrm{E}-02$ & $8 \mathrm{E}-03$ & 2.4 & R Postcentral Gyrus BA43 \\
\hline & & 56 & 0 & 22 & $1 \mathrm{E}-02$ & $1 \mathrm{E}-02$ & 2.3 & R Precentral Gyrus BA6 \\
\hline & & 54 & -44 & 16 & $1 \mathrm{E}-02$ & 1E-02 & 2.3 & R Superior Temporal Gyrus BA13 \\
\hline & & 36 & 30 & -18 & $1 \mathrm{E}-02$ & $1 \mathrm{E}-02$ & 2.3 & R Inferior Frontal Gyrus BA47 \\
\hline & & 56 & -4 & 24 & $1 \mathrm{E}-02$ & $1 \mathrm{E}-02$ & 2.3 & R Precentral Gyrus BA6 \\
\hline & & 40 & 20 & -2 & $1 \mathrm{E}-02$ & $1 \mathrm{E}-02$ & 2.3 & R Insula \\
\hline & & 54 & 36 & 14 & $1 \mathrm{E}-02$ & $1 \mathrm{E}-02$ & 2.3 & R Middle Frontal Gyrus BA46 \\
\hline & & 30 & 14 & 12 & $1 \mathrm{E}-02$ & $1 \mathrm{E}-02$ & 2.3 & R Claustrum \\
\hline & & 18 & 6 & 8 & $1 \mathrm{E}-02$ & $1 \mathrm{E}-02$ & 2.3 & R Putamen \\
\hline & & 38 & -2 & 12 & $1 \mathrm{E}-02$ & $1 \mathrm{E}-02$ & 2.2 & R Insula BA13 \\
\hline & & 64 & -30 & -4 & $1 \mathrm{E}-02$ & $1 \mathrm{E}-02$ & 2.2 & R Middle Temporal Gyrus BA21 \\
\hline & & 42 & 2 & -2 & 1E-02 & $1 \mathrm{E}-02$ & 2.2 & R Claustrum \\
\hline & & 40 & 2 & 6 & $1 \mathrm{E}-02$ & 1E-02 & 2.2 & R Claustrum \\
\hline & & 52 & -22 & -14 & $1 \mathrm{E}-02$ & $2 \mathrm{E}-02$ & 2.2 & R Superior Temporal Gyrus BA22 \\
\hline & & 40 & 32 & -16 & $1 \mathrm{E}-02$ & 2E-02 & 2.2 & R Inferior Frontal Gyrus BA47 \\
\hline & & 66 & -42 & -24 & $1 \mathrm{E}-02$ & 2E-02 & 2.2 & R Inferior Temporal Gyrus BA20 \\
\hline & & 18 & 12 & 10 & 1E-02 & 2E-02 & 2.1 & R Caudate Body \\
\hline & & 12 & 10 & -8 & 1E-02 & 2E-02 & 2.1 & R Caudate Head \\
\hline & & 36 & -8 & 12 & $1 \mathrm{E}-02$ & 2E-02 & 2.1 & R Claustrum \\
\hline & & 50 & -12 & 26 & $1 \mathrm{E}-02$ & 2E-02 & 2.1 & R Precentral Gyrus BA6 \\
\hline & & 18 & 14 & -4 & $1 \mathrm{E}-02$ & 2E-02 & 2.1 & R Putamen \\
\hline & & 36 & 22 & -16 & 1E-02 & 2E-02 & 2.0 & R Inferior Frontal Gyrus BA47 \\
\hline & & 68 & -44 & -14 & $1 \mathrm{E}-02$ & $2 \mathrm{E}-02$ & 2.0 & R Middle Temporal Gyrus BA20 \\
\hline & & 46 & 38 & 14 & 1E-02 & 2E-02 & 2.0 & R Middle Frontal Gyrus BA46 \\
\hline & & 50 & 46 & 18 & 9E-03 & 2E-02 & 2.0 & R Middle Frontal Gyrus BA46 \\
\hline & & 42 & -48 & -12 & 9E-03 & 2E-02 & 2.0 & R Fusiform Gyrus BA37 \\
\hline & & 68 & -38 & -12 & 9E-03 & $3 \mathrm{E}-02$ & 1.9 & R Middle Temporal Gyrus BA21 \\
\hline & & 60 & -8 & -18 & 9E-03 & $3 \mathrm{E}-02$ & 1.9 & R Middle Temporal Gyrus BA21 \\
\hline \multirow[t]{18}{*}{2} & 27056 & -58 & -18 & 4 & $3 \mathrm{E}-02$ & 4E-08 & 5.4 & L Superior Temporal Gyrus BA41 \\
\hline & & -52 & -6 & 2 & $3 \mathrm{E}-02$ & 2E-06 & 4.6 & L Superior Temporal Gyrus BA22 \\
\hline & & -44 & -40 & 42 & 2E-02 & $3 \mathrm{E}-05$ & 4.0 & L Supramarginal Gyrus BA40 \\
\hline & & -58 & -46 & 16 & 2E-02 & 4E-05 & 3.9 & L Superior Temporal Gyrus BA22 \\
\hline & & -54 & -26 & 38 & 2E-02 & $5 \mathrm{E}-05$ & 3.9 & L Postcentral Gyrus BA2 \\
\hline & & -58 & -30 & 14 & 2E-02 & $5 \mathrm{E}-05$ & 3.9 & L Superior Temporal Gyrus BA42 \\
\hline & & -54 & 4 & -12 & 2E-02 & 4E-04 & 3.3 & L Superior Temporal Gyrus BA38 \\
\hline & & -48 & -2 & -10 & 2E-02 & 7E-04 & 3.2 & L Superior Temporal Gyrus BA22 \\
\hline & & -56 & -36 & 22 & 2E-02 & 8E-04 & 3.2 & L Insula BA13 \\
\hline & & -66 & -24 & 30 & 2E-02 & 9E-04 & 3.1 & L Inferior Parietal Lobule BA40 \\
\hline & & -30 & -14 & -14 & 2E-02 & 9E-04 & 3.1 & L Parahippocampal Gyrus \\
\hline & & -48 & -52 & 30 & 2E-02 & 1E-03 & 3.1 & L Superior Temporal Gyrus BA39 \\
\hline & & -42 & -26 & 2 & $2 \mathrm{E}-02$ & 2E-03 & 3.0 & L Superior Temporal Gyrus BA22 \\
\hline & & -50 & -36 & 56 & 2E-02 & 2E-03 & 2.9 & L Inferior Parietal Lobule BA40 \\
\hline & & -40 & -42 & 12 & $1 \mathrm{E}-02$ & $2 \mathrm{E}-03$ & 2.8 & L Superior Temporal Gyrus BA41 \\
\hline & & -60 & -14 & 34 & 1E-02 & 2E-03 & 2.8 & L Postcentral Gyrus BA3 \\
\hline & & -20 & -16 & -16 & 1E-02 & 1E-02 & 2.3 & L Parahippocampal Gyrus BA28 \\
\hline & & -48 & -42 & 18 & $1 \mathrm{E}-02$ & 1E-02 & 2.2 & L Superior Temporal Gyrus BA13 \\
\hline
\end{tabular}


E-02

2E-02

1E-02 2E-02

1E-02

2E-02

1E-02

2E-02

1E-02 2E-02

9E-03 2E-02

9E-03 2E-02

9E-03 3E-02

3E-02 1E-07

3E-02

3E-02

3E-02

3E-02

2E-02

2E-02

$1 \mathrm{E}-02$

1E-02

1E-02

1E-02

1E-02

1E-02

$1 \mathrm{E}-02$

9E-03

2.2 L Parahippocampal Gyrus

2.2 L Parahippocampal Gyrus BA28

2.1 L Claustrum

2.1 L Superior Temporal Gyrus BA38

$2.1 \quad$ L Postcentral Gyrus BA40

2.0 L Superior Temporal Gyrus BA39

2.0 L Superior Temporal Gyrus BA22

1.9 L Inferior Parietal Lobule BA40

5.2 L Precentral Gyrus BA6

5.0 L Inferior Frontal Gyrus BA44

4.8 L Inferior Frontal Gyrus BA9

4.7 L Middle Frontal Gyrus BA6

4.4 L Precentral Gyrus BA6

3.2 L Middle Frontal Gyrus BA6

2.9 L Precentral Gyrus BA6

2.7 L Middle Frontal Gyrus BA6

2.7 L Postcentral Gyrus BA3

2.5 L Precentral Gyrus BA6

2.5 L Precentral Gyrus BA6

2.4 L Precentral Gyrus BA6

2.2 L Postcentral Gyrus BA3

2.2 L Postcentral Gyrus BA3

1.9 L Middle Frontal Gyrus BA6

3.9 R Inferior Parietal Lobule BA40

3.8 R Inferior Parietal Lobule BA40

3.6

3.5

3.4

R Precentral Gyrus BA4

R Precentral Gyrus BA4

R Postcentral Gyrus BA2

R Inferior Parietal Lobule BA40

R Inferior Parietal Lobule BA40

R Postcentral Gyrus BA2

R Inferior Parietal Lobule BA40

R Cingulate Gyrus BA24

R Sub-Gyral BA6

R Precentral Gyrus BA6

R Middle Frontal Gyrus BA6

No Gray Matter found

R Inferior Parietal Lobule BA7

R Precentral Gyrus BA6

R Superior Parietal Lobule BA7

R Middle Frontal Gyrus BA6

R Middle Frontal Gyrus BA6

R Middle Frontal Gyrus BA6

R Inferior Parietal Lobule BA40 R Precentral Gyrus BA4

\begin{tabular}{lrrrrrr} 
2. $M>$ NM: 509 foci, 59 experiments, 2189 subjects & & & & \\
\hline 1 & -58 & -18 & 4 & $3 \mathrm{E}-02$ & $8 \mathrm{E}-09$ & 5.6 \\
& -52 & -6 & 2 & $3 \mathrm{E}-02$ & $5 \mathrm{E}-07$ & 4.9 \\
& -50 & 8 & 18 & $3 \mathrm{E}-02$ & $3 \mathrm{E}-06$ & 4.5 \\
& -54 & 8 & 12 & $2 \mathrm{E}-02$ & $5 \mathrm{E}-06$ & 4.4 \\
& -48 & 4 & 26 & $2 \mathrm{E}-02$ & $5 \mathrm{E}-06$ & 4.4 \\
& -58 & -46 & 16 & $2 \mathrm{E}-02$ & $1 \mathrm{E}-05$ & 4.2 \\
& -58 & -30 & 14 & $2 \mathrm{E}-02$ & $2 \mathrm{E}-05$ & 4.1 \\
& -44 & -40 & 40 & $2 \mathrm{E}-02$ & $3 \mathrm{E}-05$ & 4.0 \\
& -54 & -26 & 36 & $2 \mathrm{E}-02$ & $7 \mathrm{E}-05$ & 3.8 \\
& -52 & 4 & 36 & $2 \mathrm{E}-02$ & $9 \mathrm{E}-05$ & 3.7 \\
& -54 & 4 & -12 & $2 \mathrm{E}-02$ & $2 \mathrm{E}-04$ & 3.6 \\
& -48 & -2 & -10 & $2 \mathrm{E}-02$ & $3 \mathrm{E}-04$ & 3.5 \\
& -66 & -24 & 30 & $2 \mathrm{E}-02$ & $4 \mathrm{E}-04$ & 3.4 \\
& -58 & -36 & 20 & $2 \mathrm{E}-02$ & $4 \mathrm{E}-04$ & 3.4 \\
& -52 & 16 & 44 & $2 \mathrm{E}-02$ & $4 \mathrm{E}-04$ & 3.4 \\
& -52 & -34 & -6 & $2 \mathrm{E}-02$ & $5 \mathrm{E}-04$ & 3.3 \\
& -48 & 2 & 50 & $2 \mathrm{E}-02$ & $9 \mathrm{E}-04$ & 3.1 \\
& -48 & -52 & 30 & $1 \mathrm{E}-02$ & $1 \mathrm{E}-03$ & 3.0 \\
& -42 & -28 & 4 & $1 \mathrm{E}-02$ & $2 \mathrm{E}-03$ & 2.8 \\
& -46 & -4 & 54 & $1 \mathrm{E}-02$ & $3 \mathrm{E}-03$ & 2.7 \\
& -40 & -6 & 56 & $1 \mathrm{E}-02$ & $4 \mathrm{E}-03$ & 2.6 \\
& -36 & -24 & 60 & $1 \mathrm{E}-02$ & $5 \mathrm{E}-03$ & 2.6 \\
-36 & -26 & 66 & $1 \mathrm{E}-02$ & $7 \mathrm{E}-03$ & 2.4 \\
& -36 & -18 & 48 & $1 \mathrm{E}-02$ & $8 \mathrm{E}-03$ & 2.4
\end{tabular}

L Superior Temporal Gyrus BA41

L Superior Temporal Gyrus BA22

L Inferior Frontal Gyrus BA9

L Inferior Frontal Gyrus BA44

L Precentral Gyrus BA6

L Superior Temporal Gyrus BA22

L Superior Temporal Gyrus BA42

L Supramarginal Gyrus BA40

L Inferior Parietal Lobule BA40

L Precentral Gyrus BA6

L Superior Temporal Gyrus BA38

L Superior Temporal Gyrus BA22

L Inferior Parietal Lobule BA40

L Insula BA13

L Middle Frontal Gyrus BA6

L Middle Temporal Gyrus BA21

L Precentral Gyrus BA6

L Superior Temporal Gyrus BA39

L Superior Temporal Gyrus BA13

L Precentral Gyrus BA6

L Precentral Gyrus BA6

L Precentral Gyrus BA4

L Postcentral Gyrus BA3

L Postcentral Gyrus BA3 


\begin{tabular}{|c|c|c|c|c|c|c|c|c|}
\hline & & -48 & 6 & -24 & $1 \mathrm{E}-02$ & 9E-03 & 2.4 & L Superior Temporal Gyrus BA38 \\
\hline & & -66 & -40 & -2 & $1 \mathrm{E}-02$ & $1 \mathrm{E}-02$ & 2.2 & L Middle Temporal Gyrus BA21 \\
\hline & & -72 & -28 & 4 & 9E-03 & $1 \mathrm{E}-02$ & 2.2 & L Superior Temporal Gyrus BA22 \\
\hline & & -60 & -42 & 0 & 9E-03 & $2 \mathrm{E}-02$ & 2.1 & L Middle Temporal Gyrus BA21 \\
\hline & & -42 & -26 & 50 & 9E-03 & $2 \mathrm{E}-02$ & 2.1 & L Postcentral Gyrus BA40 \\
\hline & & -36 & -28 & 12 & 9E-03 & $2 \mathrm{E}-02$ & 2.0 & L Transverse Temporal Gyrus BA41 \\
\hline & & -32 & -38 & 36 & $8 \mathrm{E}-03$ & 2E-02 & 2.0 & L Sub-Gyral BA40 \\
\hline \multirow[t]{28}{*}{2} & 35744 & 54 & -10 & 4 & $3 \mathrm{E}-02$ & $4 \mathrm{E}-07$ & 4.9 & R Superior Temporal Gyrus BA22 \\
\hline & & 54 & 24 & 10 & $3 \mathrm{E}-02$ & $5 \mathrm{E}-07$ & 4.9 & R Inferior Frontal Gyrus BA45 \\
\hline & & 50 & 10 & 22 & $3 \mathrm{E}-02$ & $1 \mathrm{E}-06$ & 4.7 & R Inferior Frontal Gyrus BA9 \\
\hline & & 66 & -14 & 0 & $2 \mathrm{E}-02$ & $4 \mathrm{E}-05$ & 3.9 & R Superior Temporal Gyrus BA22 \\
\hline & & 54 & 12 & 0 & $2 \mathrm{E}-02$ & $6 \mathrm{E}-05$ & 3.9 & R Precentral Gyrus BA44 \\
\hline & & 60 & -42 & -4 & $2 \mathrm{E}-02$ & 1E-04 & 3.7 & R Middle Temporal Gyrus BA21 \\
\hline & & 52 & -32 & 18 & $2 \mathrm{E}-02$ & $1 \mathrm{E}-04$ & 3.7 & R Superior Temporal Gyrus BA41 \\
\hline & & 42 & -32 & 16 & 2E-02 & $1 \mathrm{E}-04$ & 3.6 & R Superior Temporal Gyrus BA41 \\
\hline & & 58 & -36 & 24 & $2 \mathrm{E}-02$ & $4 \mathrm{E}-04$ & 3.3 & R Insula BA13 \\
\hline & & 50 & -28 & -4 & $2 \mathrm{E}-02$ & $5 \mathrm{E}-04$ & 3.3 & R Insula BA22 \\
\hline & & 58 & 0 & -6 & 2E-02 & $6 \mathrm{E}-04$ & 3.3 & R Superior Temporal Gyrus BA22 \\
\hline & & 52 & -62 & -4 & 1E-02 & $1 \mathrm{E}-03$ & 3.1 & R Inferior Temporal Gyrus BA19 \\
\hline & & 52 & -44 & 36 & $1 \mathrm{E}-02$ & $1 \mathrm{E}-03$ & 3.1 & R Supramarginal Gyrus BA40 \\
\hline & & 54 & -60 & -8 & 1E-02 & $2 \mathrm{E}-03$ & 3.0 & R Middle Occipital Gyrus BA19 \\
\hline & & 36 & 22 & 8 & $1 \mathrm{E}-02$ & $2 \mathrm{E}-03$ & 2.9 & R Insula BA13 \\
\hline & & 54 & 6 & 32 & $1 \mathrm{E}-02$ & $3 \mathrm{E}-03$ & 2.7 & R Precentral Gyrus BA6 \\
\hline & & 66 & -40 & 8 & $1 \mathrm{E}-02$ & $4 \mathrm{E}-03$ & 2.7 & R Middle Temporal Gyrus BA22 \\
\hline & & 54 & -44 & 16 & $1 \mathrm{E}-02$ & $5 \mathrm{E}-03$ & 2.6 & R Superior Temporal Gyrus BA13 \\
\hline & & 60 & -30 & 8 & 1E-02 & $5 \mathrm{E}-03$ & 2.6 & R Superior Temporal Gyrus BA41 \\
\hline & & 40 & 20 & -2 & 1E-02 & $6 \mathrm{E}-03$ & 2.5 & R Insula \\
\hline & & 52 & -22 & -14 & $1 \mathrm{E}-02$ & $8 \mathrm{E}-03$ & 2.4 & R Superior Temporal Gyrus BA22 \\
\hline & & 50 & -64 & -18 & $1 \mathrm{E}-02$ & $8 \mathrm{E}-03$ & 2.4 & R Fusiform Gyrus BA37 \\
\hline & & 64 & -30 & -6 & $1 \mathrm{E}-02$ & 9E-03 & 2.4 & R Middle Temporal Gyrus BA21 \\
\hline & & 66 & -42 & -24 & 1E-02 & $9 \mathrm{E}-03$ & 2.4 & R Inferior Temporal Gyrus BA20 \\
\hline & & 68 & -44 & -14 & $1 \mathrm{E}-02$ & $1 \mathrm{E}-02$ & 2.3 & R Middle Temporal Gyrus BA20 \\
\hline & & 34 & 22 & -8 & $1 \mathrm{E}-02$ & $1 \mathrm{E}-02$ & 2.2 & R Claustrum \\
\hline & & 40 & -40 & 24 & 9E-03 & $2 \mathrm{E}-02$ & 2.1 & R Insula BA13 \\
\hline & & 60 & -8 & -18 & 9E-03 & $2 \mathrm{E}-02$ & 2.1 & R Middle Temporal Gyrus BA21 \\
\hline \multicolumn{9}{|c|}{ 3. $N M>M: 166$ foci, 20 experiments, 591 subjects } \\
\hline \multirow[t]{2}{*}{1} & 768 & -50 & -36 & 56 & 1E-02 & $3 \mathrm{E}-05$ & 4 & L Inferior Parietal Lobule BA40 \\
\hline & & -46 & -40 & 52 & $1 \mathrm{E}-02$ & $7 \mathrm{E}-05$ & 3.8 & L Inferior Parietal Lobule BA40 \\
\hline 2 & 624 & -48 & 8 & 36 & 2E-02 & $6 \mathrm{E}-06$ & 4.4 & L Precentral Gyrus BA6 \\
\hline 3 & 232 & 48 & -34 & 66 & $2 \mathrm{E}-02$ & $3 \mathrm{E}-05$ & 4 & R Postcentral Gyrus BA2 \\
\hline 4 & 200 & 64 & -14 & 38 & $1 \mathrm{E}-02$ & $7 \mathrm{E}-05$ & 3.8 & R Precentral Gyrus BA4 \\
\hline 5 & 184 & 22 & -66 & -24 & 1E-02 & $2 \mathrm{E}-04$ & 3.6 & R Cerebellum. Declive \\
\hline 6 & 168 & 58 & 12 & 22 & 1E-02 & $2 \mathrm{E}-04$ & 3.5 & R Inferior Frontal Gyrus BA9 \\
\hline 7 & 168 & -6 & 18 & 48 & $1 \mathrm{E}-02$ & $3 \mathrm{E}-04$ & 3.4 & L Superior Frontal Gyrus BA6 \\
\hline \multirow[t]{2}{*}{8} & 160 & 18 & 6 & 10 & $1 \mathrm{E}-02$ & $5 \mathrm{E}-04$ & 3.3 & R Caudate Body \\
\hline & & 18 & 12 & 10 & 1E-02 & $7 \mathrm{E}-04$ & 3.2 & R Caudate Body \\
\hline 9 & 120 & 62 & -22 & 2 & $1 \mathrm{E}-02$ & $3 \mathrm{E}-04$ & 3.4 & R Superior Temporal Gyrus BA41 \\
\hline 10 & 104 & 38 & -60 & 50 & $1 \mathrm{E}-02$ & $3 \mathrm{E}-04$ & 3.4 & R Superior Parietal Lobule BA7 \\
\hline
\end{tabular}




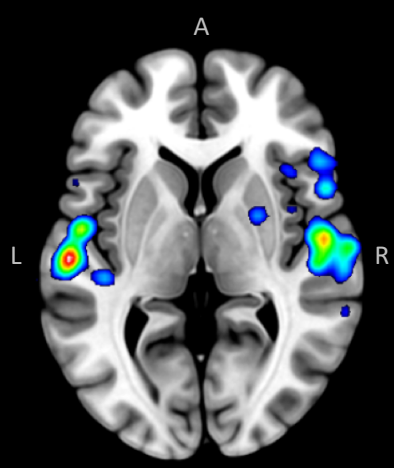

50

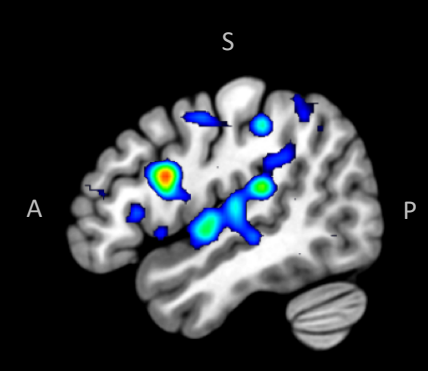

10

S

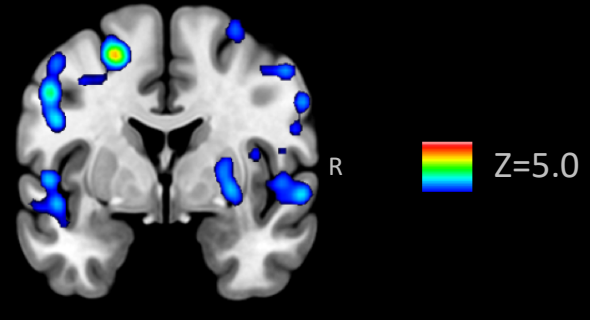

22

ALL FOCI
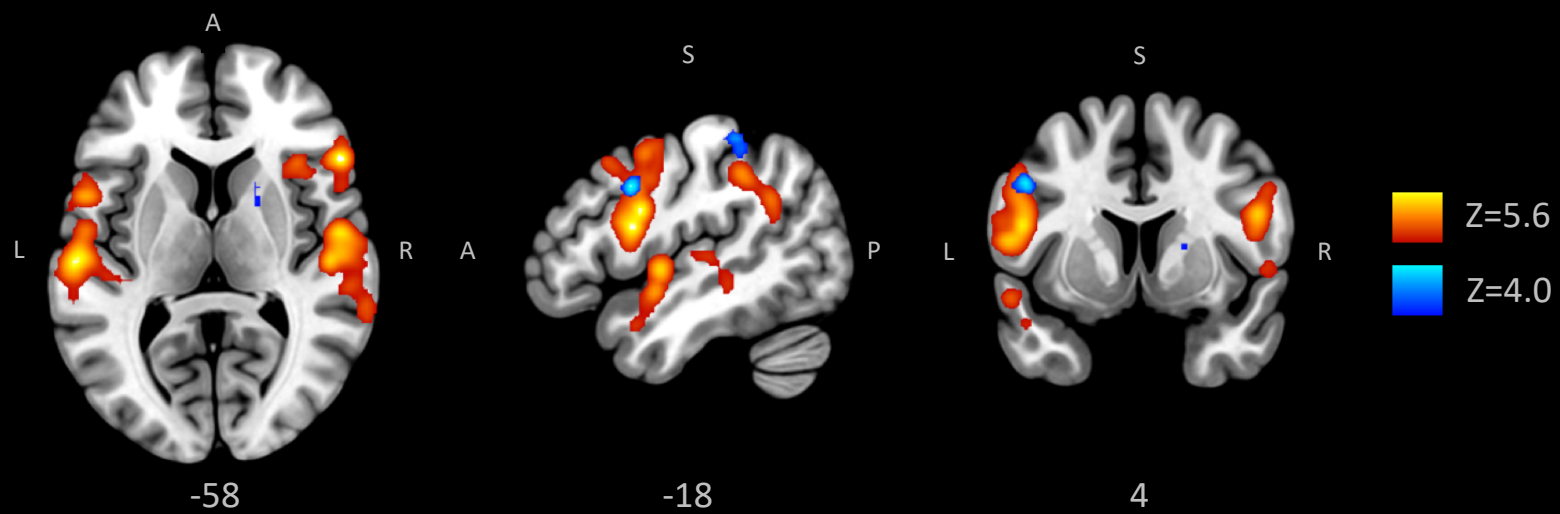

4

M vs NM
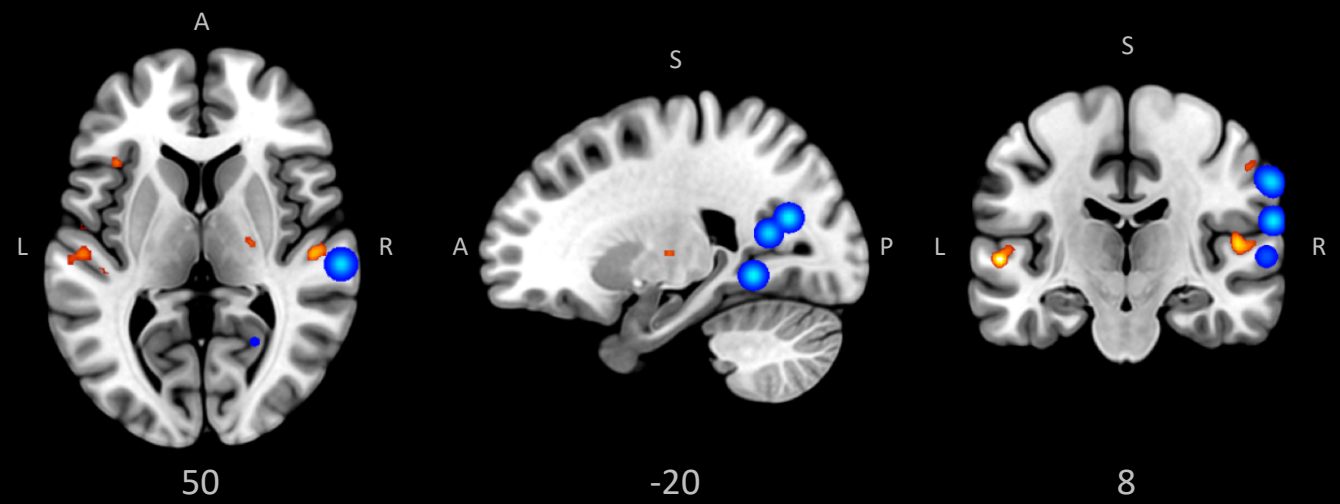

$Z=4.4$

$Z=4.7$

M vs NM (STRUCTURAL)
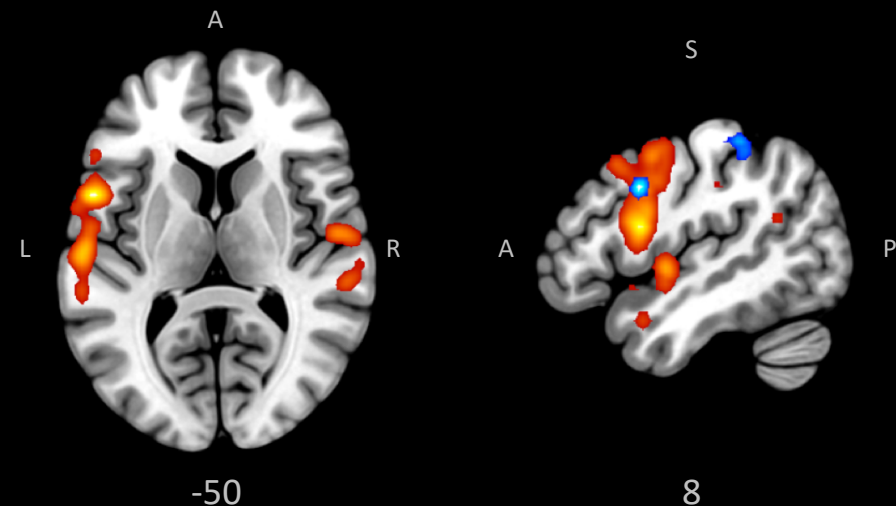

S

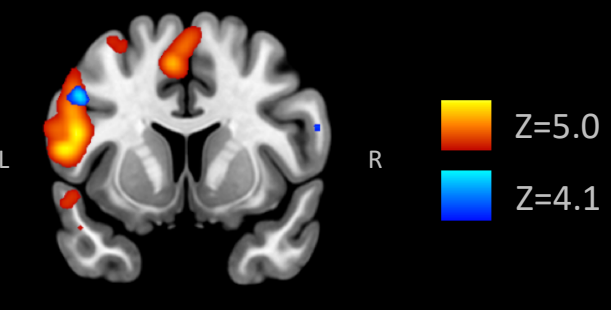

18

M vs NM (FUNCTIONAL) 


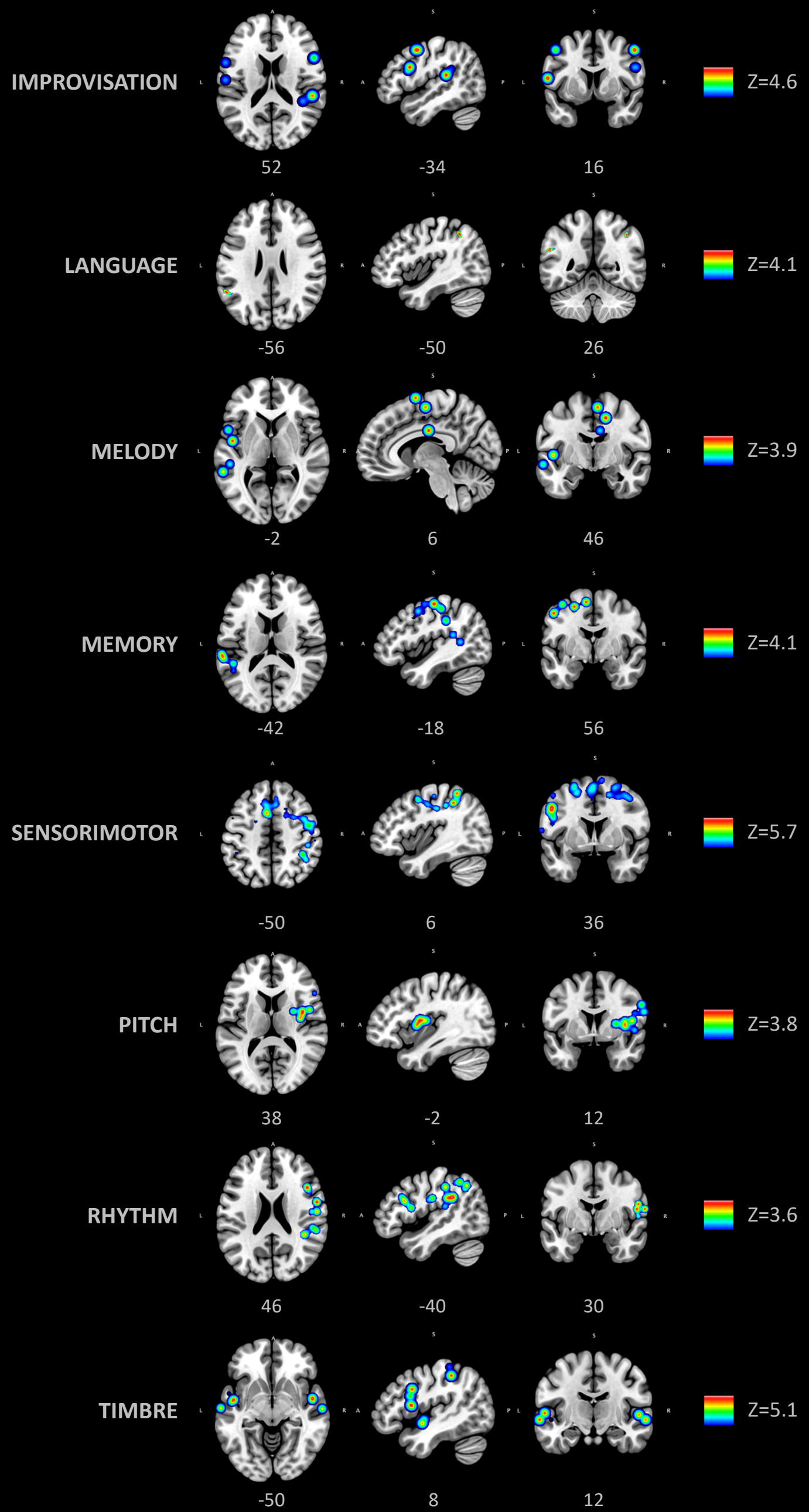

